\title{
Análisis de las respuestas cognitivas relacionadas con el diagnóstico de cáncer en pacientes mexicanos
}

\section{Cognitive response related to cancer diagnosis in Mexican patients}

\author{
Mariana A. Sierra-Murguía ${ }^{1}$, Gabriela Navarro-Contreras ${ }^{2}$, \\ Martha Leticia Guevara-Sanginés ${ }^{3}$ y Ferrán Padrós Blásquez ${ }^{4}$ \\ ${ }^{1}$ Maestra en Psicología. Psicooncóloga en Cancer Center Tec100, Universidad Michoacana San \\ Nicolás de Hidalgo, Querétaro, México. E-mail: msierra@cancercentertec100.com \\ ${ }^{2}$ Doctora en Psicología. Profesora Investigadora Titular “A” T. C. de Psicología en la Universidad \\ de Guanajuato, León, Guanajuato, México. E-mail: g.navarro@ugto.mx \\ ${ }^{3}$ Doctora en Psicología. Profesora Investigadora Titular “B”, Cuerpo Académico de \\ Comportamiento Organizacional, Universidad de Guanajuato, Guanajuato, México. \\ E-mail: leticiag@ugto.mx \\ ${ }^{4}$ Doctor en Psicología. Profesor Investigador en la Facultad de Psicología, Universidad \\ Michoacana San Nicolás de Hidalgo, Morelia, Michoacán, México. E-mail: fpadros@uoc.edu \\ Este trabajo forma parte de una investigación doctoral en Psicología de la Salud, realizada en \\ el Doctorado Interinstitucional en Psicología y cuenta con el apoyo de la beca de posgrado \\ del Consejo Nacional de Ciencia y Tecnología (CONACYT) (CVU 258666) de Chile. \\ Los autores agradecen al Consejo Nacional de Ciencia y Tecnología (CONACYT) por \\ el apoyo económico, y al doctor Juan Manuel Fraga por facilitar la realización de la \\ investigación en el Cancer Center Tec100.
}

Cancer Center Tec100. Hospital H+, Col. Centro.

Querétaro, México.

\section{Resumen}

La evaluación subjetiva ha sido descripta como una variable mediadora para la respuesta emocional. Se presenta con diferentes contenidos y estilos. El estilo cognitivo se refiere a la manera en que se piensa sobre el estresor, por ejemplo: la rumiación (pensamientos intrusivos, repetitivos, que generan malestar emocional), la evitación (intentos por no pensar en el estresor) y el compromiso cognitivo (enfocados en búsqueda de sentido o solución). En base a esta respuesta emocional, se busca explorar y describir los pensamientos relacionados con el cáncer en un grupo de diez pacientes con la enfermedad.
En un estudio cualitativo, se les pidió a diez pacientes con cáncer que indicaran los cinco pensamientos más frecuentes desde su diagnóstico. Se analizó el contenido mediante la técnica top down (teoría a contenido) y, a partir de los estilos de respuestas cognitivas, se clasificó el contenido de los pensamientos reportados por estilo de pensamiento (rumiación, compromiso cognitivo o evitación), por temporalidad (pasado, presente y futuro) y por valencia (positiva o negativa). Posteriormente, se hizo el análisis bottom up (contenido a teoría). Se hicieron tablas cruzadas para identificar los contenidos según el estilo de pensamiento, la temporalidad y la valencia. En los pensamientos con estilo rumiativo, 
el enfoque temporal se dirigía en su mayoría al futuro y al pasado, mientras que el compromiso cognitivo se enfocaba en el presente. Esta diferencia fue estadísticamente significativa mediante una prueba de chi cuadrado. Dentro de los temas relacionados con la incertidumbre, se refirieron a la muerte, al pronóstico de la enfermedad, a la respuesta de la familia y a las finanzas (en el futuro) y a las causas y culpa (en el pasado).

Palabras clave: psicooncología, respuesta cognitiva, estilo de pensamiento, rumiación, compromiso cognitivo.

\section{Abstract}

Cancer has been defined by the World Health Organization as "a disease caused by abnormal cells growing uncontrollably in the body, going beyond their usual boundaries, invading adjoining parts of the body and spreading to other organs". Being diagnosed with cancer has been described as an experience with diverse emotional reactions and difficulties in adaptation, having psychological consequences, being most common depression, anxiety and psychological distress; but also, some patients present positive consequences after having a cancer diagnosis, such as post traumatic growth, which includes positive changes presented after the disease experience. Subjective appraisal about cancer has been defined as a mediating variable for the emotional response presented after a stressor, such as cancer diagnosis, treatment, or important changes during disease process (initiating and finalizing treatment, presenting relapse or remission, as well as when having control studies). The subjective appraisal can be presented with different thought content and styles. Thought style is referred as the way thoughts about the stressor are presented, it has been classified as rumination (thoughts that are intrusive and repetitive that also increase psychological distress), cognitive engagement (thoughts associated with problem solving and meaning finding) or avoi- dance (aiming to stop thinking about stressor or feeling associated with it). Depending on the thought style presented at a stressor, could be the emotional response (anxiety, depression, distress, post-traumatic growth).

The aim of the present study was to describe the thoughts (style, content, valence and temporality) related to cancer diagnosis in a group of ten oncologic patients. To identify if differences between thought style, valence and temporality were presented.

The study design is qualitative from a phenomenological point of view. Ten oncologic (different cancer type, clinical stage and treatment) patients were asked to write the five more frequent thoughts since their cancer diagnosis. Each thought was classified by: thought style, valence, and temporality (top-down, classifying the thoughts according to theory). The same thoughts were also classified in semantic units (bottom-up, classifying the thoughts according to their own contents), generating concerns from the content. Cross tabs were made to identify contents and temporality for thought style and valence, which were analyzed by chi square to identify differences between thought style, temporality and valence. The most common thought style was cognitive engagement, followed by rumination (almost same frequency). On what respects to thought style and temporality, rumination was more focused on past (causes and attributions of cancer) and future (uncertainty about death and treatment effectiveness); meanwhile, cognitive engagement was focused on present (to enjoy present moment and focus on daily actions). Chi square was made to identify if this difference was significant, showing temporality was significantly different in cognitive engagement and rumination. The concerns identified in study participants were uncertainty about future and disease, family (communication and wellness of the family), finances (due to expensive treatments), causes and guilt about having cancer.

Thoughts that were more centered in 
present, were more likely to be associated with cognitive engagement. Cognitive engagement has been described previously as an important variable associated with post traumatic growth. Thoughts centered in the past and present were more associated with rumination. Rumination has been associated with depression and post-traumatic stress disorder. Because of mediating role of thought style in disease adaptation, emotional response to the disease, and the relation of this adaptation with the perception of quality of life in cancer patients, the design of psychological interventions aiming on promoting cognitive engagement by problem solving skills, meaning finding, and focusing on present could be a research line derived from present studies results.

Keywords: psycho-oncology, cognitive response, thought style, rumination, cognitive engagement.

\section{Introducción}

La Organización Mundial de la Salud (OMS) define el cáncer como:

"un término genérico que designa un amplio grupo de enfermedades que pueden afectar a cualquier parte del organismo (...) se caracteriza por la multiplicación rápida de células anormales que se extienden más allá de sus límites habituales y pueden invadir partes adyacentes del cuerpo o propagarse a otros órganos, proceso llamado metástasis" (OMS, 2019).

A su vez, la OMS (2019) señala al cáncer como la segunda causa de muerte a nivel mundial, siendo el causante de 8.8 millones de defunciones en el año 2015.

La experiencia del cáncer representa un estresor importante, ya que involucra un grupo de enfermedades debilitantes, crónicas, que implican un riesgo letal y que, a su vez, va acompañado de una serie de eventos aversivos como son el diagnóstico, los trata- mientos y sus efectos secundarios -náuseas, vómitos, cambios físicos como alopecia, pérdida de peso, etcétera (Bardwell y Fiorentino, 2012; Kangas, 2013). El diagnóstico y tratamiento de cáncer implica una serie de retos y situaciones adversas para el paciente que conlleva frecuentemente a experimentar estrés, malestar emocional (distrés), dificultades en el ajuste a la enfermedad y una disminución en las interacciones sociales (Classen et al., 2008). Es frecuente que, además, se presenten cambios en el funcionamiento diario, en los roles sociales, familiares y laborales, además de la incertidumbre por la posibilidad de la recurrencia y la muerte (Fallowfield y Jenkins, 2015). No todos los cambios psicológicos presentados por el cáncer son negativos o estresantes; se ha descripto que algunos pacientes presentan la percepción de cambios positivos después de un diagnóstico y tratamiento del cáncer (Ochoa Arnedo y Casellas-Grau, 2017), y que pueden dividirse en cambios espirituales, percepción de uno mismo, nuevas posibilidades en relaciones con otros y apreciación de la vida. A esta percepción de cambios positivos, surgidos después de un evento potencialmente traumático, se la llama "crecimiento postraumático" (Calhoun y Tedeschi, 2006).

Una de las variables descripta como mediadora para la respuesta emocional (positiva o negativa) y la adaptación a la enfermedad es la evaluación subjetiva que la persona hace sobre ella, a la cual se la ha relacionado de forma más intensa con el crecimiento postrauma o malestar emocional, que en medidas más objetivas como el estadío de la enfermedad (Widows, Jacobsen, Booth-Jones y Fields, 2005). Se presenta con diferentes contenidos y estilos. El estilo cognitivo se refiere a la manera en que se presentan los pensamientos sobre el estresor, mientras que el contenido se refiere a los asuntos sobre los cuales van los pensamientos y los diferentes temas. Este puede ir enfocado en temporalidad pasada, presente y futura $\mathrm{y}$, dependiendo del contenido y la respuesta emocional que presente, 
la valencia puede clasificarse en positiva o negativa. La respuesta emocional a un evento potencialmente traumático se asociará al estilo y al contenido del pensamiento, describiéndose entonces como estilos de pensamiento: evitación, compromiso cognitivo y rumiación (Shigemoto, Low, Borowa y Robitschek, 2017).

\section{Estilos de pensamiento}

El estilo de pensamiento se refiere a la manera en que se presentan pensamientos sobre el estresor, independientemente del contenido. Ha sido descripto como una característica cognitiva que tiene un efecto mediador en un evento potencialmente traumático, siendo que puede incrementar o disminuir la vulnerabilidad al TEPT y al TDM (Shigemoto et al., 2017).

Se ha estudiado en contextos patológicos, por ejemplo: rumiación en la depresión (du Pont, Hyun Rhee, Corley, Hewitt y Friedman, 2018); evitación en trastornos de ansiedad (Hofmann y Hay, 2018) y relacionados con el estrés (Taghavi y Kazemi, 2019), y se ha descripto la rumiación como un proceso de vulnerabilidad cognitiva. El estilo de pensamiento, como el compromiso cognitivo, también se presenta en contextos normativos (pensamientos asociados a posibles soluciones de un problema) (DuPre, 2017). El efecto protector del estilo de pensamiento ha sido poco estudiado (Yuan et al., 2011); es un tipo de comportamiento relativamente sencillo de entrenar pretrauma (Shigemoto et al., 2017).

\section{Rumiación}

La rumiación se refiere a pensamientos automáticos, repetitivos e intrusivos que, acorde a su mismo contenido, puede ser clasificada en rumiación deliberada (también llamada compromiso cognitivo) e intrusiva (Cann et al., 2011). Dentro del constructo de la rumiación, han sido descriptos dos factores: rumiación melancólica y reflexiva (Treynor,
González y Nolen-Hoeksema, 2003). La rumiación melancólica se conceptualiza como un estilo de pensamiento en el que se tiende a comparar el estado actual de la persona con algún estándar no logrado (por ejemplo, en el cáncer, puede presentarse como pensamientos de cosas que la persona siente que debería estar realizando en lugar de estar enfermo). La rumiación reflexiva, por otro lado, se refiere a un pensamiento enfocado en la resolución activa del problema en que se percibe la persona (Treynor et al., 2003).

\section{Compromiso cognitivo}

Debido a las diferencias en la conceptualización del término "rumiación", Smith y Alloy (2009) hacen énfasis en los diferentes propósitos que puede tener y que debe ser diferenciado, según esto, para distinguir los pensamientos repetitivos benéficos de los dañinos. En este sentido, Calhoun y Tedeschi (2006) proponen el término "compromiso cognitivo", haciendo énfasis en que al utilizar la palabra "rumiación", no se refieren a pensamientos repetidos e intrusivos, que generan malestar, sino pensamientos enfocados en el evento, dedicados a comprender y analizar una situación propositivamente. En el presente artículo, de igual manera, se utilizará el término "compromiso cognitivo", haciendo énfasis en la diferencia entre la rumiación, que tiene la característica de ser involuntaria y automática, y el compromiso cognitivo, que se refiere a pensamientos deliberados.

\section{Evitación cognitiva}

La evitación cognitiva se refiere a las estrategias internas utilizadas para evitar exponerse a un pensamiento o imagen molesta. Se mantiene debido a que, de manera inmediata, genera una disminución de la ansiedad (temporal); sin embargo, a mediano y largo plazo, impide que la persona afronte la situación estresante $\mathrm{y}$, por ende, su procesamiento emocional (Rauch y Foa, 2006). El uso de la 
evitación cognitiva previene el procesamiento mental de una experiencia estresante $y$, a largo plazo, puede incrementar los pensamientos intrusivos y el malestar emocional que se asocia a un incremento en la percepción de síntomas físicos asociados al tratamiento en pacientes con cáncer (Bauer et al., 2017). Se ha descripto una relación bidireccional entre evitación cognitiva y pensamientos intrusivos en pacientes con cáncer de mama, sobre todo en los primeros tres meses del diagnóstico, pero disminuye a los seis meses (siendo un proceso dinámico) (Bauer et al., 2017).

\section{Contenido de pensamiento}

El contenido específico del pensamiento puede predecir el ajuste a un estresor (Segerstrom et al., 2003). Una de las formas en que los pensamientos repetitivos pueden ser adaptativos es mediante la reflexión y el procesamiento emocional-funcional (Shigemoto et al., 2017); estos serán pensamientos con valencia positiva, debido al impacto que tienen en el ajuste emocional. El procesamiento se refiere a los pensamientos repetitivos que pueden aparecer tras experiencias potencialmente traumáticas o emociones negativas; sin embargo, se hace una diferenciación entre preocupación patológica $\mathrm{y}$ pensamientos resolutivos (DuPre, 2017). Las preocupaciones funcionales (no patológicas) y las intrusiones son una primera etapa para la aceptación y acomodación, seguida por pensamientos resolutivos que se caracterizan por la contemplación enfocada a la modificación de esquemas o reinterpretaciones positivas (Calhoun y Tedeschi, 2006). Este tipo de pensamientos, al presentarse después de un evento potencialmente traumático, se han asociado a encontrar sentido a este evento o crecimiento por la experiencia, por lo que este es benéfico cuando es breve y/o controlable o cuando es verbalizado o compartido con otras personas, en lugar de ser procesado de forma incontrolable por largos períodos de tiempo y en aislamiento (Segerstrom et al., 2003).
En el modelo de crecimiento postraumático propuesto por Calhoun y Tedeschi (2006), se utiliza el término "compromiso cognitivo", haciendo énfasis en que es un pensamiento repetitivo, que no necesariamente es intrusivo y que incluye recuerdos, búsqueda de solución de problemas e intentos por encontrar un sentido a la situación estresante (Shigemoto et al., 2017). Por ello, es importante conocer las características y el contenido del pensamiento asociado a un evento potencialmente traumático.

El crecimiento postraumático se ha correlacionado positivamente con el compromiso cognitivo, ya que este proceso permite reestructurar las creencias sobre sí mismo y el mundo, además de encontrar un sentido al evento que se está viviendo (Calhoun y Tedeschi, 2006). La finalidad es que se logre una comprensión del fenómeno, pues, al lograrlo, se incrementan las posibilidades de afrontamiento y se llega a la conclusión de que la persona cuenta con recursos suficientes para lidiar con ello. Estos dos primeros aspectos del compromiso cognitivo con el trauma son acordes a la evaluación primaria -evaluar un suceso como amenazante- y secundaria evaluar los recursos personales para su afrontamiento-, propuestas por Lazarus y Folkman (Lazarus, 1993).

\section{Contenido de los pensamientos en pacientes con cáncer}

Respecto a los contenidos de los pensamientos, específicamente en el contexto oncológico, existe un concepto relacionado con la evaluación cognitiva, y es la incertidumbre por la enfermedad, que ha sido descripto como un fenómeno frecuente en estos pacientes. La incertidumbre proviene de situaciones ambiguas, complicadas e impredecibles (Wright et al., 2009). Específicamente, la incertidumbre relacionada con la enfermedad se asocia a situaciones estresantes causadas por condiciones médicas, además de variables personales y sociales, como la calidad de la infor- 
mación, creencias previas de la enfermedad, conocimiento de ella (Wright et al., 2009).

Dentro del contenido de las rumiaciones pueden incluirse entonces pensamientos relacionados con esta incertidumbre, temas como la ambigüedad e impredictibilidad del curso o pronóstico de la enfermedad, temas relacionados con la complejidad de los tratamientos y la falta de información relacionada con el tratamiento y la enfermedad. A su vez, esta incertidumbre por la enfermedad se ha asociado a una mayor respuesta de estrés y malestar emocional (Shigemoto et al., 2017). Estas serán tomadas como valencia negativa debido al impacto negativo que generan en la adaptación emocional al cáncer.

En un estudio realizado con el objetivo de evaluar la adecuación de un modelo de Psicoterapia Centrada en la Compasión (PCC), se exploró el contenido de los pensamientos en un grupo de pacientes con cáncer terminal. Los temas identificados fueron los relacionados con la enfermedad y el tratamiento, la compasión / autocompasión, la aceptación, base segura, meditación, tareas, síntomas físicos, estado emocional, sentido, recursos, miedos / preocupaciones, cambios a partir de la enfermedad, relación y comunicación con otros significativos (Prieto Larroya et al., 2017). Estos temas también son clasificados en contenido de pensamiento.

Debido al papel que tiene el estilo y contenido del pensamiento ante un evento estresante, como el diagnóstico de cáncer, el presente estudio tiene como objetivo explorar y describir los pensamientos relacionados con la enfermedad en un grupo de pacientes mexicanos con diagnóstico oncológico, conforme a los estilos de pensamiento, la temporalidad, la valencia y el contenido temático que comportan. Existe un estudio que describe el contenido temático de pensamientos en pacientes con cáncer (Prieto Larroya et al., 2017), realizado en población española; como no ha sido estudiado en población mexicana, es un aporte de la presente investigación. Conocer el estilo de pensamiento y su contenido puede ayudar a entender las respuestas emocionales de los pacientes con cáncer y a diseñar intervenciones enfocadas en ellos para promover una mayor adaptación a la enfermedad.

\section{Método}

Es un estudio transversal, con alcance descriptivo, corte fenomenológico y componentes cuantitativos. El muestreo es intencional no probabilístico, con metodología cualitativa y técnicas de recolección de datos a través de un cuestionario abierto (Kerlinger y Lee, 2002).

\section{Participantes}

La muestra estuvo compuesta por diez pacientes oncológicos de diagnóstico reciente (no mayor a tres meses), que se encontraban recibiendo tratamiento con quimioterapia en una clínica oncológica privada en la ciudad de Querétaro, México. El muestreo fue por conveniencia y todos los pacientes participaron de manera voluntaria y anónima. De los diez pacientes, seis eran mujeres y el promedio de edad fue de 42 años, con un rango de 36-58 años. Los diagnósticos de los pacientes fueron: cinco cáncer de mama, dos cáncer de vejiga, dos de próstata y un cervicouterino.

\section{Instrumento}

Para el presente estudio se utilizó la siguiente consigna: "Mencione los cinco pensamientos más frecuentes desde su diagnóstico".

\section{Aspectos éticos}

Todos los participantes aceptaron participar de manera voluntaria y firmaron consentimiento informado. El protocolo fue sometido al comité de ética e investigación del hospital donde se encuentra la clínica. 


\section{Procedimiento}

En la sala de espera del centro oncológico se abordó de manera personal a los pacientes, se les explicó de qué se trataba el estudio, cuáles eran los objetivos y se procedió con la firma del consentimiento informado. Se les pidió a los participantes que escribieran los cinco pensamientos más frecuentes que hubieran presentado a raíz de su diagnóstico. Se hicieron tres análisis: el primero de la teoría al contenido (top-down); el segundo del contenido a la teoría (bottom-up), y el tercero, tablas de contingencia para describir los contenidos por estilo de pensamiento, temporalidad y valencia, a cuya distribución se le aplicó la prueba de independencia de chi cuadrado para conocer si existían asociaciones significativas del estilo de pensamiento con la temporalidad y la valencia.

\section{Análisis de datos}

\section{Primer análisis (top-down)}

En este primer análisis de contenido, se leyeron las aseveraciones anotadas por los pacientes y, por cada pensamiento reportado, el investigador lo clasificó según la variable de interés en las siguientes categorías:

1) Estilo de pensamiento: rumiación, compromiso cognitivo o evitación

2) Temporalidad: pasado, presente $o$ futuro

3) Valencia: positiva o negativa

\section{Segundo análisis (bottom-up)}

En esta clasificación, se partió de cada pensamiento independiente del análisis anterior, reportado por los pacientes, y el investigador identificó de manera exhaustiva las unidades semánticas conforme a los temas a los que aludieran. Estos temas sirvieron para describir el contenido de los pensamientos.

\section{Tercer análisis (tablas de contingencia)}

Se hicieron tablas de contingencia para identificar los contenidos según el estilo de pensamiento (rumiación, compromiso cognitivo o evitación), la valencia (positiva o negativa) y la temporalidad (pasado, presente y futuro). Esta clasificación se hizo por dos de los investigadores de manera separada $\mathrm{y}$, si había desacuerdos en la clasificación de algún pensamiento, se comentaban entre ambos investigadores para llegar a un acuerdo.

Posteriormente, se realizó una prueba de chi cuadrado para conocer si existían diferencias en temporalidad y valencia según el estilo de pensamiento. En este análisis no se incluyó evitación, ya que el instrumento pedía ejemplos de pensamientos que presentaran, no que evitaran, por lo que no se consideró pertinente incluirlo.

\section{Resultados}

De los 50 pensamientos reportados, se identificaron 95 unidades semánticas, clasificadas en 16 temas. Los más frecuentes fueron: incertidumbre, familia, aceptación, muerte y optimismo. Algunos pensamientos reportados presentaban combinaciones de más de un tema; por ejemplo, incertidumbre y muerte: " ¿Qué pasará? ¿Moriré?”, incertidumbre y familia: “¿Qué pasará con mi familia?”. Dentro del tema de incertidumbre, se identificaron pensamientos como "¿Quedaré igual que antes?" o "¿Y si no funciona el tratamiento?". Dentro del tema familia, había temas relacionados con la incertidumbre de ya no estar con sus seres queridos, por la posibilidad de morir y afectar su bienestar, ya sea en el presente ("No quiero causarles sufrimiento") o en el futuro, relacionado con la muerte e incertidumbre ("Si muero, no sé qué será de ellos"). Dentro del tema de aceptación, se incluían pensamientos como "Son cosas que pasan", "Hay que vivir el día a día", y algunos incluían, además de la aceptación, la religión, por ejemplo: "Me pongo en manos de Dios, que 
sea su voluntad", "Dios nos manda todo para nuestro bien".

En el análisis de estilo de pensamiento, se identificaron 46 pensamientos del estilo de compromiso cognitivo (enfocados en búsqueda de sentido, solución de problemas), se incluyeron pensamientos del tipo "Pienso muy a menudo en disfrutar del presente de cada día, cada detalle y agradecer que tengo la oportunidad de vivirlo y llegar a mi futuro próximo que es el mañana y disfrutarlo de igual manera". En este pensamiento podemos identificar el compromiso cognitivo, con temporalidad presente y futura. Otro pensamiento identificado como compromiso cognitivo es "Me debo esforzar para estar sana nuevamente". En cuanto al estilo rumiativo de pensamiento, se identificaron 45 que incluyeron pensamientos como tristeza, preocupación, "Pienso ¿Por qué a mí, si no fumo, no tomo y soy deportista?”, “¿Por qué me dio cáncer a mí?”, "Debí haber sido mamá". Por último, en la respuesta cognitiva del tipo evitativo, se incluyeron pensamientos del tipo "Cuando algún pensamiento negativo o triste pasa por mi mente, trato al máximo de cambiarlo por algún pensamiento positivo $\mathrm{y}$ me apoyo en la meditación, oración y música, así no pienso en eso", "Me repito frases positivas como 'todo saldrá bien"”.

En cuanto a la temporalidad del pensamiento (pasado, presente y futuro), de las 95 unidades semánticas, 43 se encontraban en el presente, 33 en el pasado y 19 en el futuro. Algunos ejemplos de pensamientos enfocados en el presente fueron: "Tratar de disfrutar las cosas, aunque haya dolor o cansancio", "Aceptar el cambio en mi cuerpo". En pensamientos asociados al pasado, se incluyeron "Aunque estaba al pendiente, no lo pude detectar a tiempo", "No hice lo que hubiera querido en la vida".

En cuanto al futuro, algunos ejemplos

"Si sobrevivo, seré invencible", "¿Y si no funcionan los tratamientos?".

En cuanto a la valencia de los pensamientos, se identificaron 45 con valencia negativa, incluyendo pensamientos como "No hice todo en la vida", “¿Por qué a mí? ¿Qué hice para merecerlo?", "No quiero que mis hijos sufran por mi culpa". En pensamientos con valencia positiva, se identificaron 50 y se incluyeron pensamientos como "Tengo muchas ganas de vivir", "Debo aprovechar el día a día".

La tipificación de cada unidad semántica presentada por los pacientes, de acuerdo a la clasificación por estilo de pensamiento, temporalidad, valencia y contenido, se muestra en el Anexo 1.

La distribución de los pensamientos expresados por estilo de pensamiento y temporalidad se muestra en la Tabla 1. Existe una mayor cantidad de pensamientos enfocados en el presente cuando existe compromiso cognitivo y más enfocados en el pasado o el futuro cuando existe rumiación, en tanto que hay pocos casos de evitación. Se muestra una relación estadísticamente significativa entre la temporalidad y el estilo del pensamiento, según la cual el compromiso cognitivo está más enfocado en el presente y la rumiación, hacia el pasado y el futuro $\left(\chi^{2}=13.884 \mathrm{gl}=2\right.$, $\mathrm{p}=.001)$.

\section{Tabla 1}

Estilo de pensamiento por temporalidad.

\begin{tabular}{c|c|c|c|c} 
Estilo de pensamiento & Pasado & Presente & Futuro & Total \\
\hline Compromiso cognitivo & 4 & 28 & 13 & 45 \\
Evitación & & 3 & 1 & 4 \\
Rumiación & 15 & 12 & 19 & 46 \\
Total & 19 & 43 & 33 & 95
\end{tabular}


El análisis de evocaciones por valencia y temporalidad (Tabla 2) indica una relación estadísticamente significativa entre ambas $\left(\chi^{2}\right.$ $=14.309 \mathrm{gl}=2, \mathrm{p}=.001)$. Los pensamientos son mayormente positivos cuando se enfocan en el presente y más negativos hacia el pasado, mientras que los enfocados hacia el futuro son tanto positivos como negativos.

Tabla 2

Temporalidad del pensamiento por su valencia.

\begin{tabular}{c|c|c|c} 
Temporalidad & Negativa & Positiva & Total \\
\hline Pasado & 14 & 5 & 19 \\
Presente & 10 & 30 & 40 \\
Futuro & 18 & 14 & 32 \\
Total & 42 & 49 & 91
\end{tabular}

Se identificaron 16 temas en relación con el contenido de los pensamientos (Tabla 3). En la temporalidad en el futuro, en primer lugar se identificó la incertidumbre, seguida por la muerte y, en tercer lugar, la familia. En cuanto a la temporalidad presente, en primer lugar se presentó el valorar la vida/optimismo (un ejemplo, "Tengo muchas ganas de vivir"); en segundo lugar, el tema de la aceptación, con pensamientos como "Pienso muy a menudo en disfrutar del presente de cada día, cada detalle y agradecer que tengo la oportunidad de vivirlo y llegar a mi futuro próximo, que es el mañana, y disfrutarlo de igual manera"; en tercer lugar, el tema identificado fue la

emoción, que incluyó pensamientos como "Actitud, fe y esperanza, pueden librar de cualquier enfermedad". Para la temporalidad pasada, en primer lugar autoindulgencia/ culpa, con pensamientos como "Preocupación por mis familiares al saber que llevan una carga por mi culpa". Como siguiente tema, se encontró la aceptación, "Efectivamente, al recibir el diagnóstico, me impresioné, después estuve en paz”; las causas “¿Por qué me dio a mí? ¿Qué hice mal?” y, por último, el tema recurrente "deberías", que incluyó pensamientos como "Debí haber sido mamá", "No hice todo lo que hubiera querido en la vida" (Anexo 1).

Tabla 3

Temas / Contenido del pensamiento por temporalidad.

\begin{tabular}{c|c|c|c|c} 
Asunto & Pasado & Presente & Futuro & Total \\
\hline Valor vida/ optimismo & 1 & 11 & 3 & 15 \\
Incertidumbre & 1 & 2 & 10 & 13 \\
Agradecimiento & & 2 & & 2 \\
Autoindulgencia / culpa & 6 & 2 & & 8 \\
Familia & 1 & 2 & 5 & 8 \\
Muerte & & & 6 & 6 \\
Emoción & 1 & 4 & 1 & 6 \\
Religión & 1 & 3 & 1 & 5 \\
Sentido & & 3 & 2 & 5 \\
Crecimiento & 1 & 2 & 2 & 5 \\
Lucha & & 2 & 2 & 4
\end{tabular}




\begin{tabular}{c|c|c|c|c} 
Asunto & Pasado & Presente & Futuro & Total \\
\hline Debí & 2 & & 1 & 3 \\
Tratamiento & 1 & 1 & & 2 \\
Finanzas & & 1 & & 1 \\
Total & 15 & 35 & 33 & 83
\end{tabular}

\section{Discusión}

Los objetivos del presente estudio fueron explorar y describir el estilo, la valencia, la temporalidad y el contenido de los pensamientos asociados al diagnóstico de cáncer en una muestra de pacientes oncológicos. El estilo de pensamiento ha sido clasificado, por otros autores, según su propósito (solución $v s$. búsqueda de problema) y concluyen en que el contenido, más que el estilo de pensamiento, se relacionaría con la respuesta emocional, que se asocia con la depresión, la ansiedad, la ira, las conductas de salud y los niveles de estrés (Smith y Alloy, 2009).

En la muestra que se estudió, aproximadamente la mitad de los pensamientos iban enfocados en generar una reevaluación cognitiva, es decir, búsqueda de sentido y enfocada a solución de problemas; la otra mitad estaba enfocada en la rumiación. Sin embargo, no se identificaron pensamientos relacionados con la evitación; esto puede ser debido a que se les pidió que describieran pensamientos frecuentes y no pensamientos que trataban de evitar. Las diferencias presentadas entre los estilos de pensamiento identificados (rumiación y compromiso cognitivo) fueron la temporalidad y su valencia.

Se identificó que en los pensamientos con estilo rumiativo, el enfoque temporal se dirigía, en su mayoría, al futuro (relacionado con la incertidumbre por el desenlace del tratamiento y de la enfermedad) y al pasado (buscando causas y culpas por el diagnóstico), mientras que el compromiso cognitivo estuvo orientado hacia el presente. Estos resultados coinciden con los reportados por McLaughlin y Nolen-Hoeksema (2011) con respecto a la temporalidad del pensamiento, quienes describen que el estilo rumiativo se modifica a lo largo del tiempo: se inicia con pensamientos enfocados en el pasado, pero evoluciona hacia un enfoque al futuro. Estos resultados podrían explicar por qué se identificaban hacia el pasado y el futuro cuando el estilo de pensamiento era rumiativo.

En cuanto a la valencia, también se encontró una diferencia significativa en la temporalidad, ya que los pensamientos enfocados en el presente tenían, predominantemente, valencia positiva (asociados al compromiso cognitivo), mientras que los pensamientos enfocados en el pasado presentaban valencia negativa (asociada a la rumiación); para el futuro se dividían en rumiación o compromiso cognitivo, dependiendo del contenido del pensamiento. Al presentarse rumiación con enfoque al futuro, se identificaron temas como incertidumbre y temor, contrarios al compromiso cognitivo enfocado al futuro que se presentaba con planeación de actividades al terminar el tratamiento y cambios positivos asociados a cambios en valores personales, más importancia a relaciones personales, espirituales, bienestar personal, etcétera. Esto se identificó en la presente investigación: el estilo de pensamiento de rumiación se enfocaba, en su mayoría, en el futuro (incertidumbre sobre qué pasará) y en el pasado enfocado en las causas, generando malestar emocional.

El concepto de "incertidumbre a la enfermedad" ha sido descripto en pacientes oncológicos y es definido como un estresor cognitivo, que incluye una sensación de pérdida de control y percepción de duda que se modifica a lo largo del tiempo; es relevante, pues se asocia a dificultades en el ajuste a la enfermedad, afrontamiento desadaptativo, mayores niveles de malestar emocional y una reducción 
en la calidad de vida (Wright et al., 2009).

Uno de los elementos que ha sido considerado importante en el contenido de los pensamientos es la discrepancia entre el estado actual y el estado deseado, ya que el cuestionamiento sobre las causas o el por qué existe esta discrepancia es uno de los temas principales que se encuentran en el contenido de la rumiación (Watkins, 2008). Esto fue identificado en la muestra estudiada mediante pensamientos intrusivos que cuestionan las causas de la enfermedad o por qué le sucede a ellos. Estos pensamientos se asocian al malestar emocional y no se enfocan en resolver esta discrepancia o el malestar que genera, a diferencia del compromiso cognitivo, en el que, a pesar de identificar esta discrepancia, los pensamientos se enfocan en solucionarlos o en resolver la emoción generada.

En cuanto al contenido, se identificó con mayor prevalencia la incertidumbre, específicamente relacionada al futuro (desenlace del tratamiento y enfermedad) y solamente una relacionada con el pasado (relacionada con las causas de la enfermedad). Dentro de los temas relacionados con la incertidumbre en la población de estudio, esta fue asociada a la posibilidad de muerte, al pronóstico de la enfermedad, a la respuesta de la familia y a las finanzas (en el futuro), así como a las causas y culpa (en el pasado).

Como siguiente tema del contenido de los pensamientos se encuentra valor de la vida/ optimismo. Este tipo de contenidos, contrario a lo que sucede con la incertidumbre, se asocia al crecimiento postrauma, que se refiere a la percepción de cambios positivos ocurridos después de una situación potencialmente traumática (Prieto Larroya et al., 2017). Este modelo plantea cinco componentes del crecimiento postrauma: cambios en la percepción de uno mismo, nuevas posibilidades, relaciones con otros, espiritualidad y cambios en la apreciación de la vida (Calhoun y Tedeschi, 2006). Dentro del tema de valor a la vida y optimismo, identificado en la población de estudio, se describen verbalizaciones y pensamientos que hacen alusión a estos componentes, que no se asocian al malestar emocional.

El proceso de pensar atenta, repetida o frecuentemente, sobre uno mismo y su contexto es un elemento central en diferentes modelos de ajuste (adaptativo o desadaptativo) (Segerstrom et al., 2003). Existen diferentes visiones sobre este proceso: una de ellas es que los pensamientos repetitivos interfieren con la capacidad de solución de problemas y mejorías en el estado anímico, mientras que otra es que pueden generar cambios positivos en la evaluación cognitiva, la búsqueda de sentido y las soluciones a los mismos problemas. Es por ello que se han propuesto diferentes dimensiones relacionadas con este tipo de pensamientos (Segerstrom et al., 2003).

Se logró describir y explorar el estilo, el contenido y la temporalidad de los pensamientos asociados al diagnóstico de cáncer en esta población. Los hallazgos del presente estudio son exploratorios y consistentes con la teoría presentada.

Una de las limitaciones del estudio es que se realizó sobre una muestra pequeña, de pacientes tratados en una clínica privada (homogénea en nivel socioeconómico y educativo medio-alto), por lo que los resultados no son generalizables al resto de la población. Sin embargo, cumplieron el objetivo de describir e identificar los estilos de pensamiento presentados y las diferencias en cuanto al contenido, la temporalidad y la valencia.

Se resalta la importancia del estilo de pensamiento, que brinda un primer acercamiento a sus contenidos, su valencia y su temporalidad, en pacientes con cáncer. Debido al papel mediador que tiene el estilo de pensamiento en la respuesta de adaptación a la enfermedad y, a su vez, la relación de esto con la calidad de vida en el paciente con cáncer, el diseño de intervenciones psicológicas encaminadas a promover el compromiso cognitivo mediante el enfoque en el presente, la búsqueda de sentido y la solución de problemas, puede ser una vertiente de la misma línea de inves- 
tigación. Dentro de las terapias psicológicas que han mostrado evidencia para promover el enfoque en el presente, la búsqueda de sentido y/o la solución de problemas en pacientes con cáncer, se destacaron la Psicoterapia Positiva para Pacientes con Cáncer (PPC) (Ochoa et al., 2017), el Mindfulness-based cancer recovery (Carlson, 2013), la psicoterapia centrada en la compasión para pacientes con cáncer (PCC) (Prieto Larroya et al., 2017), la intervención bioconductual para pacientes con cáncer (Andersen et al., 2009), la terapia de aceptación y compromiso para pacientes con cáncer (Hulbert-Williams y Wilson, 2015) y la terapia cognitivo conductual adyuvante para paciente con cáncer (Greer, 2008).

Se sugiere diseñar instrumentos con propiedades psicométricas adecuadas para medir estilos de pensamiento y contenido en pacientes oncológicos. Los resultados del presente estudio pueden ser de utilidad para diseñar las dimensiones y los ítems de este instrumento, tomando en cuenta los estilos de pensamiento y cómo es que se presenta cada uno en cuanto a la temporalidad, la valencia y los asuntos.

Por último, se sugiere un estudio cuantitativo descriptivo con una muestra mayor y prospectivo, para poder determinar la relación del estilo de pensamiento con la respuesta emocional ante el diagnóstico y tratamiento, e identificar el papel que tiene la respuesta cognitiva (los estilos de pensamiento y su contenido) en afrontar el cáncer y su tratamiento. Esto, a su vez, justificaría que se diseñaran tratamientos psicológicos breves y enfocados en promover el crecimiento postrauma y una mayor adaptación al cáncer mediante el compromiso cognitivo y el enfoque en el presente.

\section{Referencias}

Andersen, B. L., Golden-Kreutz, D. M., Emery, C. F. y Thiel, D. L. (2009). Biobehavioral Intervention for Cancer Stress: Conceptualization, Components, and Intervention Strat- egies. Cognitive and Behavioral Practice, 16(3), 253-265. https://doi.org/10.1016/j. cbpra.2008.11.002

Bauer, M. R., Wiley, J. F., Weihs, K. L. y Stanton, A. L. (2017). Stuck in the spin cycle: Avoidance and intrusions following breast cancer diagnosis. British Journal of Health Psychology, 22(3), 609-626. https://doi.org/10.1111/ bjhp. 12252

Bardwell, W. A. y Fiorentino, L. (2012). Risk factors for depression in breast cancer survivors: An update. International Journal of Clinical and Health Psychology, 12(2), 311-331. https://psycnet.apa.org/record/2012-11670009

Calhoun, L. G. y Tedeschi, R. G. (2006). Handbook of posttraumatic growth: Research and Practice. Lawrence Erlbaum Associates, Publihers.

Cann, A., Calhoun, L. G., Tedeschi, R. G., Triplett, K. N., Vishnevsky, T. y Lindstrom, C. M. (2011). Assessing posttraumatic cognitive processes: The event related rumination inventory. Anxiety, Stress and Coping, 24(2), 137-156. https://doi.org/10.1080/10615806.2 010.529901

Carlson, L. E. (2013). Mindfulness-based cancer recovery. The development of an evidencebased psychosocial oncology intervention. Oncology Exchange, 12(2), 21-25.

Classen, C. C., Kraemer, H. C., Blasey, C., Giese-Davis, J., Koopman, C., Palesh, O. G., ... Spiegel, D. (2008). Supportive-expressive group therapy for primary breast cancer patients: a randomized prospective multicenter trial. Psycho-Oncology, 17(5), 438-447. https://doi.org/10.1002/pon.1280

du Pont, A., Hyun Rhee, S., Corley, R., Hewitt, J. K. y Friedman, N. P. (2018). Rumination and Psychopathology: Are Anger and Depressive Rumination Differentially Associated with Internalizing and Externalizing Psychopathology? Clinical Psychological Science, 6(1), 18-31. https://doi. org/10.1177/2167702617720747

DuPre E. y Spreng N. (2017). Spontaneous thoughts gets stuck with rumination. En The 
Oxford Handbook of Spontaneous Thought: Mind Wandering, creativity, dreaming and clinical contitions. Eds. Fox K. y Christoff K.

Fallowfield, L. y Jenkins, V. (2015). Psychosocial/ survivorship issues in breast cancer: Are we doing better? Journal of the National Cancer Institute, 107(1), 1-5. https://doi.org/10.1093/ jnci/dju335

Greer, S. (2008). CBT for emotional distress of people with cancer: some personal observations, 173(May 2007), 170-173. https://doi. org/10.1002/pon.1205

Hofmann, S. G. y Hay, A. C. (2018). Rethinking avoidance: Toward a balanced approach to avoidance in treating anxiety disorders. Journal of Anxiety Disorders, 55, 14-21. https://doi. org/10.1016/j.janxdis.2018.03.004

Hulbert-Williams, N. J. y Wilson, K. G. (2015). Psychological interventions for patients with cancer: psychological flexibility and the potential utility of Acceptance and Commitment Therapy. European Journal of Cancer Care, 24, 15-27. https://doi.org/10.1111/ecc.12223

Kangas, M. (2013). DSM-5 trauma and stress-related disorders: Implications for screening for cancer-related stress. Frontiers in Psychiatry, 4(OCT), 2-4. https://doi.org/10.3389/ fpsyt.2013.00122

Kerlinger, F. y Lee, H. (2002). Investigación del comportamiento. México: McGraw Hill/ Interamericana.

Lazarus, R. S. (1993). Coping theory and research: past, present, and future. Psychosomatic Medicine, 55(3), 234-247. http://www.ncbi.nlm. nih.gov/pubmed/8346332

McLaughlin, K. A. y Nolen-Hoeksema, S. (2011). Rumination as a transdiagnostic factor in depression and anxiety. Behaviour Research and Therapy, 49(3), 186-193. https://doi. org/10.1016/j.brat.2010.12.006

Ochoa-Arnedo, C. y Casellas-Grau, Y. A. (2017). Positive life change after cancer: The key ingredients to facilitate it and effects on wellbeing. Papeles Del Psicologo, 38(1), 1-11. https://doi.org/10.23923/pap.psicol2017.2815

Ochoa- Arnedo, C., Casellas-Grau, A., Vives, J., Font, A. y Borràs, J. M. (2017). Psicote- rapia Positiva para supervivientes de cáncer con elevados niveles de malestar emocional: la facilitación del crecimiento postraumático reduce el estrés postraumático. International Journal of Clinical and Health Psychology, 17(1), 28-37. https://doi.org/10.1016/j. ijchp.2016.09.002

OMS (2019). Nota descriptiva sobre el cáncer. https://www.who.int/topics/cancer/es/

Prieto Larroya, C., Chamorro López, J., Fernández Castro, J. y Gil Moncayo, F. L. (2017). Qualitative analysis of constructed and emerging themes from a pilot experience of Compassion Centered Psychotherapy (PCC) in patients with advanced cancer. Psicooncología, 14(1), 149-158. https://doi.org/10.5209/PSIC.55818

Rauch, S. y Foa, E. (2006). Emotional processing theory (EPT) and exposure therapy for PTSD. Journal of Contemporary Psychotherapy, 36(2), 61-65. https://doi.org/10.1007/s10879006-9008-y

Segerstrom, S. C., Stanton, A. L., Alden, L. E. y Shortridge, B. E. (2003). A Multidimensional Structure for Repetitive Thought: What's on Your Mind, and How, and How Much? Journal of Personality and Social Psychology, 85(5), 909-921. https://doi.org/10.1037/00223514.85.5.909

Shigemoto, Y., Low, B., Borowa, D. y Robitschek, C. (2017). Function of Personal Growth Initiative on Posttraumatic Growth, Posttraumatic Stress, and Depression Over and Above Adaptive and Maladaptive Rumination. Journal of Clinical Psychology, 73(9), 1126-1145. https://doi.org/10.1002/jclp.22423

Smith, J. M. y Alloy, L. B. (2009). A roadmap to rumination: a review of the definition, assessment, and conceptualization of this multifaceted construct. Clinical Psychology Review, 29(2), 116-128. https://doi.org.10.1016/j. cpr.2008.10.003

Taghavi, R. y Kazemi, R. (2019). Effectiveness of brief behavioral activation treatment on the rumination and experiential avoidance in veterans with post-traumatic stress disorder. Iranian Journal of War and Public Health, 11(1), 1-6. 
Treynor, W., Gonzalez, R. y Nolen-Hoeksema, S. (2003). Rumination Reconsidered: a psychometric analysis. Cognitive Therapy and Research, 27(3), 247-259. https://doi.org/10.1023/A https://doi. org/10.1023/A:1023910315561

Watkins, E. R. (2008). Constructive and Unconstructive Repetitive Thought. Psychological Bulletin, 134(2), 163-206. https://doi. org/10.1037/0033-2909.134.2.163

Widows, M. R., Jacobsen, P. B., Booth-Jones, M. y Fields, K. K. (2005). Predictors of Posttraumatic Growth Following Bone Marrow Transplantation for Cancer. Health Psychology,
24(3), 266-273. https://doi.org/10.1037/02786133.24.3.266

Wright, L. J., Afari, N. y Zautra, A. (2009). The illness uncertainty concept: A review. Current Pain and Headache Reports, 13(2), 133-138. https://doi.org/10.1007/s11916-009-0023-Z

Yuan, C., Wang, Z., Inslicht, S., McCaslin, S., Metzler, T., Henn-Haase, C., ... Marmar, C. (2011). Protective factors for posttraumatic stress disorder symptoms in a prospective study of police officers. Psychiatry Research, 188(1), 45-50. https://doi.org.10.1016/j. psychres.2010.10.034

Recibido: 14 de mayo de 2020 Aceptado: 30 de septiembre de 2021 


\section{Anexo 1}

Tabla de contingencias para clasificar estilo de pensamiento, temporalidad, tema y ejemplo de pensamiento.

\begin{tabular}{|c|c|c|c|c|}
\hline $\begin{array}{c}\text { Estilo de } \\
\text { pensamiento }\end{array}$ & Temporalidad & Tema & Pensamiento & Valencia \\
\hline \multirow[t]{17}{*}{$\begin{array}{l}\text { Compromiso } \\
\text { cognitivo }\end{array}$} & \multirow[t]{13}{*}{ Futuro } & \multirow[t]{2}{*}{ Crecimiento } & $\begin{array}{l}\text { Esperanza: a salir adelante para superar esta } \\
\text { situación y que me sirva de experiencia }\end{array}$ & Positiva \\
\hline & & & Si sobrevivo, seré invencible & Positiva \\
\hline & & $\begin{array}{l}\text { Disfrutar } \\
\text { presente }\end{array}$ & $\begin{array}{l}\text { Pienso muy a menudo en disfrutar del } \\
\text { presente de cada día, cada detalle y agra- } \\
\text { decer que tengo la oportunidad de vivirlo } \\
\text { y llegar a mi futuro próximo que es el } \\
\text { mañana y disfrutarlo de igual manera }\end{array}$ & Positiva \\
\hline & & Familia & $\begin{array}{c}\text { ¿Qué sigue? ¿Qué voy a hacer? ¿Y mis } \\
\text { papás? }\end{array}$ & Negativa \\
\hline & & \multirow[t]{2}{*}{ Incertidumbre } & ¿Qué procede? & Negativa \\
\hline & & & Si sobrevivo, seré invencible & Positiva \\
\hline & & Lucha & $\begin{array}{l}\text { Pensamientos relacionados a posibles solu- } \\
\text { ciones, tratamientos y avances científicos }\end{array}$ & Positiva \\
\hline & & Muerte & Si sobrevivo, seré invencible & Positiva \\
\hline & & Optimismo & $\begin{array}{l}\text { Esperanza: a salir adelante para superar esta } \\
\text { situación y que me sirva de experiencia }\end{array}$ & Positiva \\
\hline & & Religión & $\begin{array}{l}\text { Le pido a Dios que me dé salud, Él siempre } \\
\text { está, ha estado y estará con nosotros }\end{array}$ & Positiva \\
\hline & & \multirow[t]{2}{*}{ Sentido } & $\begin{array}{l}\text { Me debo esforzar para estar sana nueva- } \\
\text { mente }\end{array}$ & Positiva \\
\hline & & & $\begin{array}{l}\text { Pienso muy a menudo en disfrutar del } \\
\text { presente de cada día, cada detalle y agra- } \\
\text { decer que tengo la oportunidad de vivirlo } \\
\text { y llegar a mi futuro próximo que es el } \\
\text { mañana y disfrutarlo de igual manera }\end{array}$ & Positiva \\
\hline & & Valor vida & $\begin{array}{c}\text { Aprendizaje: pienso en que puede servir } \\
\text { esta situación para aprender y mejorar mi } \\
\text { vida }\end{array}$ & Positiva \\
\hline & \multirow[t]{4}{*}{ Pasado } & Aceptación & $\begin{array}{l}\text { Efectivamente, al recibir el diagnóstico, me } \\
\text { impresioné, después estuve en paz }\end{array}$ & Positiva \\
\hline & & Crecimiento & Tuve que tener cáncer para valorar la vida & Positiva \\
\hline & & Emoción & $\begin{array}{l}\text { Efectivamente, al recibir el diagnóstico, me } \\
\text { impresioné, después estuve en paz }\end{array}$ & Positiva \\
\hline & & Religión & $\begin{array}{l}\text { Le pido a Dios que me dé salud, Él siempre } \\
\text { está, ha estado y estará con nosotros }\end{array}$ & Positiva \\
\hline
\end{tabular}


Sierra-Murguía, Navarro-Contreras, Guevara-Sanginés y Padrós Blásquez

\begin{tabular}{|c|c|c|c|c|}
\hline $\begin{array}{c}\text { Estilo de } \\
\text { pensamiento }\end{array}$ & Temporalidad & Tema & Pensamiento & Valencia \\
\hline \multirow{5}{*}{$\begin{array}{c}\text { Compromiso } \\
\text { cognitivo } \\
\text { (cont. })\end{array}$} & \multirow[t]{5}{*}{ Presente } & \multirow[t]{4}{*}{ Aceptación } & Aceptar el cambio en mi cuerpo & Positiva \\
\hline & & & Aprende uno a vivir el día a día & Positiva \\
\hline & & & Dios nos manda todo para nuestro bien & Positiva \\
\hline & & & $\begin{array}{l}\text { Estoy en las manos de Dios y estoy } \\
\text { dispuesta a que se haga su voluntad }\end{array}$ & Positiva \\
\hline & & Agradecimiento & $\begin{array}{l}\text { El amor de tu familia se hace notar con } \\
\text { mucha fuerza, es algo muy bonito }\end{array}$ & Positiva \\
\hline
\end{tabular}

Pienso muy a menudo en disfrutar del presente de cada día, cada detalle y agradecer que tengo la oportunidad de vivirlo

Positiva

y llegar a mi futuro próximo que es el mañana y disfrutarlo de igual manera

\begin{tabular}{|c|c|c|}
\hline Cambio & Aceptar el cambio en mi cuerpo & Positiva \\
\hline \multirow[t]{2}{*}{ Crecimiento } & $\begin{array}{l}\text { Actitud, fe y esperanza, pueden vibrar de } \\
\text { cualquier enfermedad }\end{array}$ & Positiva \\
\hline & $\begin{array}{l}\text { Aprendizaje: pienso en que puede servir esta } \\
\text { situación para aprender y mejorar mi vida }\end{array}$ & Positiva \\
\hline \multirow{4}{*}{$\begin{array}{l}\text { Disfrutar } \\
\text { presente }\end{array}$} & Aprende uno a vivir el día a día & Positiva \\
\hline & $\begin{array}{c}\text { Pienso en mis hobbies, en lo que disfruto } \\
\text { hacer y en proyectos que me gustaría } \\
\text { realizar }\end{array}$ & Positiva \\
\hline & $\begin{array}{l}\text { Pienso muy a menudo en disfrutar del } \\
\text { presente de cada día, cada detalle y agra- } \\
\text { decer que tengo la oportunidad de vivirlo } \\
\text { y llegar a mi futuro próximo que es el } \\
\text { mañana y disfrutarlo de igual manera }\end{array}$ & Positiva \\
\hline & $\begin{array}{l}\text { Tratar de disfrutar las cosas, aunque haya } \\
\text { dolor o cansancio }\end{array}$ & Positiva \\
\hline Emoción & $\begin{array}{l}\text { Actitud, fe y esperanza, pueden vibrar de } \\
\text { cualquier enfermedad }\end{array}$ & Positiva \\
\hline Familia & $\begin{array}{c}\text { El amor de tu familia se hace notar con } \\
\text { mucha fuerza, es algo muy bonito }\end{array}$ & Positiva \\
\hline \multirow[t]{2}{*}{ Lucha } & Debo luchar & Positiva \\
\hline & $\begin{array}{l}\text { Me debo esforzar para estar sana nueva- } \\
\text { mente }\end{array}$ & Positiva \\
\hline \multirow[t]{3}{*}{ Optimismo } & $\begin{array}{l}\text { Actitud, fe y esperanza, pueden vibrar de } \\
\text { cualquier enfermedad }\end{array}$ & Positiva \\
\hline & $\begin{array}{l}\text { Me debo esforzar para estar sana nueva- } \\
\text { mente }\end{array}$ & Positiva \\
\hline & $\begin{array}{l}\text { Tratar de disfrutar las cosas, aunque haya } \\
\text { dolor o cansancio }\end{array}$ & Positiva \\
\hline
\end{tabular}




\begin{tabular}{|c|c|c|c|c|}
\hline $\begin{array}{c}\text { Estilo de } \\
\text { pensamiento }\end{array}$ & Temporalidad & Tema & Pensamiento & Valencia \\
\hline \multirow{8}{*}{$\begin{array}{l}\text { Compromiso } \\
\text { cognitivo } \\
\text { (cont.) }\end{array}$} & \multirow[t]{8}{*}{ Presente (cont.) } & \multirow[t]{3}{*}{ Religión } & Dios nos manda todo para nuestro bien & Positiva \\
\hline & & & $\begin{array}{l}\text { Estoy en las manos de Dios y estoy } \\
\text { dispuesta a que se haga su voluntad }\end{array}$ & Positiva \\
\hline & & & $\begin{array}{l}\text { Vivir es un regalo de Dios, si me acerco a } \\
\text { Él, todo es más fácil }\end{array}$ & Positiva \\
\hline & & \multirow[t]{3}{*}{ Sentido } & $\begin{array}{l}\text { Le pido a Dios que me dé salud, Él siempre } \\
\text { está, ha estado y estará con nosotros }\end{array}$ & Positiva \\
\hline & & & $\begin{array}{c}\text { Tratar de disfrutar las cosas, aunque haya } \\
\text { dolor o cansancio }\end{array}$ & Positiva \\
\hline & & & $\begin{array}{l}\text { Vivir es un regalo de Dios, si me acerco a } \\
\text { él, todo es más fácil }\end{array}$ & Positiva \\
\hline & & Tratamiento & $\begin{array}{l}\text { Pensamientos relacionados a posibles solu- } \\
\text { ciones, tratamientos y avances científicos }\end{array}$ & Positiva \\
\hline & & Valor vida & $\begin{array}{l}\text { Pienso en mis hobbies, en lo que disfruto } \\
\text { hacer y en proyectos que me gustaría realizar }\end{array}$ & Positiva \\
\hline \multirow[t]{2}{*}{ Evitación } & Futuro & Emoción & $\begin{array}{l}\text { Ser conciente que puedo morir si no tiene } \\
\text { éxito mi tratamiento }\end{array}$ & Negativa \\
\hline & Presente & Emoción & $\begin{array}{l}\text { Cuando algún pensamiento negativo o triste } \\
\text { pasa por mi mente, trato al máximo de } \\
\text { cambiarlo por algún pensamiento positivo } \\
\text { y me apoyo en la meditación, oración y } \\
\text { música }\end{array}$ & Negativa \\
\hline
\end{tabular}

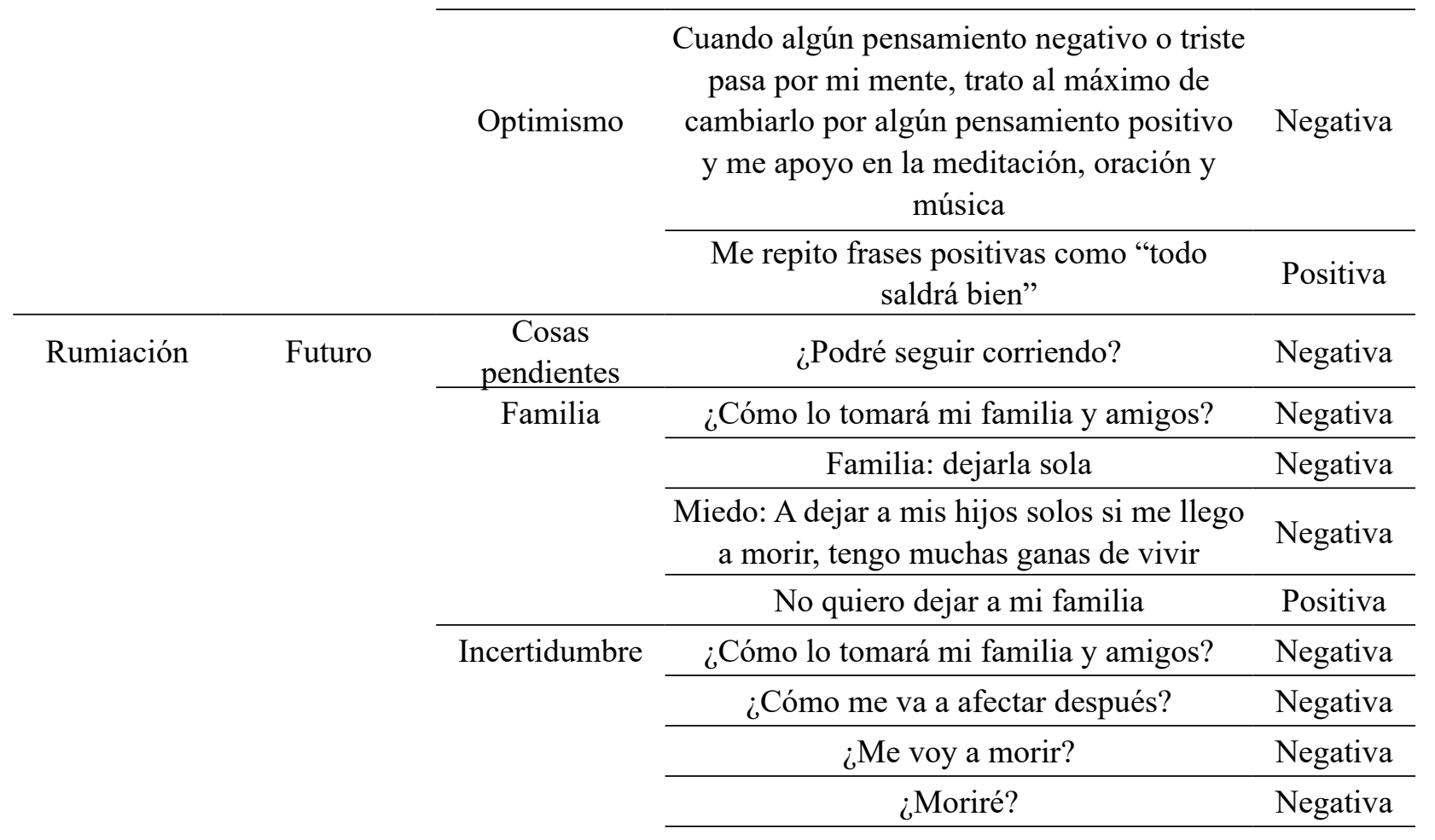


Sierra-Murguía, Navarro-Contreras, Guevara-Sanginés y Padrós Blásquez

\begin{tabular}{|c|c|c|c|c|}
\hline $\begin{array}{c}\text { Estilo de } \\
\text { pensamiento }\end{array}$ & Temporalidad & Tema & Pensamiento & Valencia \\
\hline \multirow{12}{*}{$\begin{array}{l}\text { Rumiación } \\
\text { (cont.) }\end{array}$} & \multirow[t]{10}{*}{ Futuro (cont.) } & \multirow{4}{*}{$\begin{array}{l}\text { Incertidumbre } \\
\quad(\text { cont. })\end{array}$} & $\begin{array}{c}\text { ¿Qué sigue? ¿Qué voy a hacer? ¿y mis } \\
\text { papás? }\end{array}$ & Negativa \\
\hline & & & ¿Tendré cura? & Negativa \\
\hline & & & ¿Voy a quedar igual que siempre? & Negativa \\
\hline & & & Espero quedar bien & Positiva \\
\hline & & Lucha & $\begin{array}{l}\text { Miedo: A dejar a mis hijos solos si me llego } \\
\text { a morir, tengo muchas ganas de vivir }\end{array}$ & Positiva \\
\hline & & \multirow[t]{5}{*}{ Muerte } & ¿Me voy a morir? & Negativa \\
\hline & & & ¿Moriré? & Negativa \\
\hline & & & Me voy a morir & Negativa \\
\hline & & & $\begin{array}{l}\text { Miedo: A dejar a mis hijos solos si me llego } \\
\text { a morir, tengo muchas ganas de vivir }\end{array}$ & Negativa \\
\hline & & & No quiero dejar a mi familia & Negativa \\
\hline & \multirow[t]{2}{*}{ Pasado } & \multirow{2}{*}{$\begin{array}{l}\text { Autoindul- } \\
\text { gencia }\end{array}$} & No hice todo en la vida & Negativa \\
\hline & & & $\begin{array}{l}\text { Nunca pensé que me iban a quitar todo el } \\
\text { seno }\end{array}$ & Negativa \\
\hline
\end{tabular}

Previamente había leído un mensaje en inglés en el consultorio del doctor, y en mi mente estaba la palabra "storm"... Now I am the storm

Tristeza, pues aunque estaba al pendiente, no lo pude detectar a tiempo (el cáncer)

\begin{tabular}{ccc}
\hline Causas & ¿Por qué me dio cáncer? & Negativa \\
\cline { 2 - 3 } Culpa & Causas ¿Por qué me dio? & Negativa \\
\cline { 2 - 3 } & $\begin{array}{c}\text { Tristeza, pues aunque estaba al pendiente } \\
\text { no lo pude detectar a tiempo (el cáncer) }\end{array}$ & Negativa \\
\hline $\begin{array}{c}\text { Cosas } \\
\text { pendientes }\end{array}$ & Debí haber sido mamá & Negativa \\
\cline { 2 - 3 } & No hice todo en la vida & Negativa \\
\hline Familia & Debí haber sido mamá & Negativa \\
\hline Incertidumbre & Causas ¿Por qué me dio? & Negativa \\
\hline \multirow{2}{*}{ Presente } & $\begin{array}{c}\text { Desde que me sentí la bolita, sabía que era } \\
\text { algo malo. Cuando el doctor confirmó el } \\
\text { diagnóstico, pensé “lo sabía” }\end{array}$ & Negativa \\
\hline Tratamiento & $\begin{array}{c}\text { Nunca pensé que me iban a quitar todo el } \\
\text { seno }\end{array}$ & Negativa \\
\hline Valor vida & Tuve que tener cáncer para valorar la vida & Positiva \\
\hline
\end{tabular}


https://doi.org/10.16888/interd.2022.39.1.9

\begin{tabular}{|c|c|c|c|c|}
\hline $\begin{array}{c}\text { Estilo de } \\
\text { pensamiento }\end{array}$ & Temporalidad & Tema & Pensamiento & Valencia \\
\hline \multirow[t]{12}{*}{$\begin{array}{l}\text { Rumiación } \\
\text { (cont.) }\end{array}$} & \multirow[t]{12}{*}{ Presente } & Aceptación & $\begin{array}{l}\text { Son cosas que pasan, no se sabe por qué, } \\
\text { solo pasan }\end{array}$ & Positiva \\
\hline & & $\begin{array}{c}\text { Autoindul- } \\
\text { gencia }\end{array}$ & ¿Por qué a mí? & Negativa \\
\hline & & \multirow[t]{2}{*}{ Causas } & ¿Por qué a mí? & Negativa \\
\hline & & & $\begin{array}{l}\text { Tristeza: preocupación, pienso ¿por qué a } \\
\text { mí? si no fumo, no tomo y soy deportista }\end{array}$ & Negativa \\
\hline & & Culpa & $\begin{array}{l}\text { Preocupación por mis familiares al saber } \\
\text { que llevan una carga por mi culpa }\end{array}$ & Negativa \\
\hline & & Familia & $\begin{array}{l}\text { Preocupación por mis familiares al saber } \\
\text { que llevan una carga por mi culpa }\end{array}$ & Negativa \\
\hline & & Finanzas & ¿El dinero? & Negativa \\
\hline & & \multirow[t]{2}{*}{ Incertidumbre } & $\begin{array}{l}\text { Son cosas que pasan, no se sabe por qué, } \\
\text { solo pasan }\end{array}$ & Negativa \\
\hline & & & $\begin{array}{l}\text { Tristeza: preocupación, pienso ¿por qué a } \\
\text { mí? si no fumo, no tomo y soy deportista }\end{array}$ & Negativa \\
\hline & & \multirow[t]{2}{*}{ Tristeza } & $\begin{array}{l}\text { Tristeza, pues aunque estaba al pendiente, } \\
\text { no lo pude detectar a tiempo (el cáncer) }\end{array}$ & Negativa \\
\hline & & & $\begin{array}{l}\text { Tristeza: preocupación, pienso ¿por qué a } \\
\text { mí? si no fumo, no tomo y soy deportista }\end{array}$ & Negativa \\
\hline & & Valor vida & $\begin{array}{l}\text { Miedo: A dejar a mis hijos solos si me llego } \\
\text { a morir, tengo muchas ganas de vivir }\end{array}$ & Positiva \\
\hline
\end{tabular}

